\title{
Refuerzo vertebral percutáneo: vertebroplastia y cifoplastia. Procedimiento técnico
}

\author{
J.V. Martínez-Quiñones; J. Aso-Escario y R. Arregui-Calvo
}

Servicio de Neurocirugía. Hospital MAZ. Zaragoza

\section{Resumen}

Las técnicas de refuerzo vertebral, vertebroplastia y cifoplastia, consisten básicamente en la introducción de un cemento óseo -polimetilmetacrilato (PMMA)- en un cuerpo vertebral fracturado, para aliviar el dolor mediante la estabilización de la fractura vertebral. En ocasiones, previamente a la administración del cemento, a nivel de la vértebra colapsada, se inserta un balón que se insufla con la finalidad de restaurar la altura del cuerpo vertebral y reducir la deformidad cifótica; en estos casos hablamos de cifoplastia o de vertebroplastia mediante balón.

Las indicaciones de la vertebroplastia y cifoplastia han ido evolucionando, pasando de la estabilización de una fractura vertebral osteoporótica dolorosa, al refuerzo de un colapso vertebral secundario a metástasis.

En el presente trabajo se revisan los principales aspectos técnicos de la vertebroplastia y de la cifoplastia, a la luz de nuestra experiencia en el tratamiento de las fracturas vertebrales.

Se necesitan más estudios que permitan definir el papel de cada procedimiento dentro del arsenal terapéutico de la cirugía de columna.

PALABRAS CLAVE: Vertebroplastia. Cifoplastia. Refuerzo vertebral. Trauma.

Percutaneous vertebral augmentation: vertebroplasty and kyphoplasty. Operative technique

\section{Summary}

The intravertebral injection of acrylic resin cement -usually polymethylmethacrylate (PMMA)- into a fractured vertebral body, constitutes the basis of the so called "vertebral augmentation techniques", "vertebroplasty" and "kyphoplasty", to manage pain and to

Recibido: 20-11-04. Aceptado: 10-01-05 strengthen and stabilize the compromised vertebra.

In some ocassion, prior to the PMMA injection, an inflatable bone tamp was inserted into both pedicles of the fractured vertebra with the aim of restoring vertebral height to correct the kyphosis deformation. This procedure is called kyphoplasty (balloon-assisted vertebroplasty).

The indications for vertebroplasty and kyphoplasty are evolving, from stabilization of painful osteoporotic vertebral fractures to vertebral collapse secondary to spinal metastases.

In this paper we review the technical basis of both procedures, according to our experience in the treatment of vertebral fractures. Further studies are required to define the role for each techniques in the spinal surgery's armamentarium.

KEY WORDS: Vertebroplasty. Kyphoplasty. Vertebral augmentation. Trauma.

Introducción

Durante los últimos años se ha venido produciendo en todos los campos de la Medicina un mayor interés por las técnicas mínimamente invasivas. La cirugía de la columna vertebral no se ha mantenido ajena a este creciente interés. Así lo demuestra el que se hayan desarrollado o puesto a punto técnicas como la discectomía lumbar percutánea, la discoplastia, la anuloplastia, la fusión percutánea o el refuerzo vertebral ${ }^{92}$. La vertebroplastia y la cifoplastia se incluyen entre las últimas. Dependerá de una adecuada selección de los casos que estos procedimientos perduren o no en el tiempo.

Las fracturas vertebrales en compresión constituyen un gran problema, con aproximadamente 700.000 nuevos casos por año en los Estados Unidos de América, de las cuales 280.000 causan un dolor intenso y 150.000 requie-

Abreviaturas. EVDS: European vertebral osteoporosis group. PMMA: polimtilmetracrilato. STIR: short tau inversion recovery. VP: vertebroplastia percutánea. 
ren ingreso hospitalario. Este tipo de fractura es la habitual entre los afectos de osteoporosis ${ }^{84}$. Es más, un $25 \%$ de las mujeres norteamericanas por encima de los 50 años sufrirán una o más fracturas vertebrales en compresión secundaria a osteoporosis ${ }^{33,72,103}$.

Según el estudio EVOS, en la población europea la fractura secundaria a osteoporosis más frecuente es la vertebral, existiendo al menos una fractura en el $20,2 \%$ de las mujeres y en el $12 \%$ de los varones ${ }^{87}$. Los datos en España son similares a lo comunicado por el estudio EVOS ${ }^{89}$.

De forma tradicional, el dolor originado por las fracturas vertebrales de origen osteoporático se trata sintomáticamente, alternando el reposo en cama (para disminuir el dolor) con la actividad (para evitar la aparición de nuevas fracturas osteoporóticas), el uso de analgésicos (los cuales podían causar confusión mental o depresión, sobre todo en ancianos) y de ortesis externas en hiperextensión, como las de Jewet, o la cruciforme anterior, que frecuentemente resultan mal toleradas y restringen la movilidad diafragmática. A todo esto se asocia como tratamiento específico los difosfonatos, que tardan casi un año en resultar efectivos y que tienen poca efectividad en el dolor, o la calcitonina por vía nasal, que suele resultar más efectiva para el dolor, pero menos para controlar la osteoporosis. Sólo de forma excepcional, en aquellos casos en los que una fractura se asociaba a un compromiso neurológico o a una inestabilidad espinal se planteaba un tratamiento quirúrgico.

Por otro lado, es un hecho que las fracturas vertebrales de causa osteoporótica inducen una progresiva cifosis dorsal, que puede incrementar la mortalidad de un 23 a un $35 \%$, disminuyen la capacidad vital respiratoria en un $9 \%$ por cada vértebra dorsal fracturada, aceleran la sensación de plenitud postprandial, e incrementan el grado de depresión. Muchos de estos pacientes se tornan dependientes y pueden quedar relegados en un futuro, no muy lejano para ellos, a una silla de ruedas o una cama ${ }^{6,38,56,79,85,88,94}$.

Las técnicas quirúrgicas instrumentadas no suelen indicarse en estos pacientes, incluso ante una deformidad progresiva espinal, ante el gran número de complicaciones a las que se asocian (desenroscado de los tornillos, migración de material, pérdida de estabilidad, etc.) ${ }^{9}$.

En otras ocasiones el trastorno doloroso asienta en procesos que inducen una osteolisis de un soma vertebral. Entre ellas destacan las metástasis vertebrales, originadas en orden de frecuencia decreciente en la mama, el pulmón, la próstata, el riñón y el tiroides. Otros procesos menos frecuentes serían los hemangiomas vertebrales agresivos, los mielomas o los linfomas ${ }^{98}$. Aunque estas afecciones pueden aparecer en cualquier edad, suelen ser más habituales en ancianos, afectos a su vez de múltiples procesos y con un delicado estado de salud. En estos casos suelen descartarse procedimientos quirúrgicos mayores, utilizando en ellos medidas farmacológicas, oncológicas, fisioterapéuticas u ortésicas. Las técnicas de refuerzo vertebral tendrían aquí un papel que, además, permitirían su combinación con quimio o radioterapia ${ }^{100}$.

Los pilares básicos del tratamiento de cualquier fractura son la restauración de la anatomía, la corrección de la deformidad y la preservación de la función. Conseguir estos fines, sin excesivos riesgos o agresividad quirúrgica en la fracturas vertebrales por osteoporosis, era algo impensable hasta hace poco ${ }^{79}$. En los últimos años se han desarrollado técnicas percutáneas guiadas por radioscopia (vertebroplastia y cifoplastia), con la finalidad de mitigar el dolor y reducir, en la medida de lo posible, el grado de acuñamiento vertebral, aspecto con el que se busca disminuir las consecuencias deletéreas que una deformidad vertebral podría inducir tanto en la función pulmonar como en la digestiva ${ }^{77,90}$.

La vertebroplastia fue por primera vez desarrollada en Francia (1987) para el tratamiento de angiomas vertebrales sintomáticos $^{29}$ extendiéndose posteriormente al resto de países. Once años más tarde, Reiley desarrolló en Estados Unidos, la cifoplastia ${ }^{92}$.

Estos procedimientos percutáneos, que se realizan a través de diferentes abordajes, se vienen practicando tanto por radiólogos intervencionistas ${ }^{29,48}$, traumatólogos ${ }^{5,33}$, como por neurocirujanos ${ }^{7,26}$. La vertebroplastia percutánea (VP) consistiría en la introducción de un cemento óseo -poli metilmetacrilato (PMMA)- en un cuerpo vertebral fracturado, para aliviar el dolor mediante el refuerzo y la estabilización de la fractura vertebral, sin que se reexpanda el colapso vertebral. Como la VP, la cifoplastia percutánea introduce un cemento óseo -PMMA- en el interior de un cuerpo vertebral colapsado. Sin embargo, previamente a la administración del cemento, a nivel de la vértebra colapsada, se inserta un balón que se insufla, con la finalidad de restaurar la altura del cuerpo vertebral y reducir la deformidad cifótica. Al retirar el balón quedaría en el interior del cuerpo vertebral una cavidad o nido que permitiría introducir el cemento a menor presión y con mayor viscosidad, con lo que se reduciría el riesgo de extravasación. Algunos autores prefieren denominar a la cifoplastia como "vertebroplastia mediante balón"9,73. Cabría hacerse dos preguntas: ¿Cuándo deberíamos plantearnos el uso de una técnica de refuerzo vertebral? ¿Hasta dónde son útiles estos procedimientos?

\section{Indicaciones y contraindicaciones}

Las indicaciones de estos procedimientos han ido evolucionando. En un principio se pensó que el paciente ideal era aquel que padecía dolor secundario a una fractura, que persistía por encima de las 4-6 semanas, con un grado de acuñamiento no superior al $60 \%$ de la altura vertebral, y 
con la confirmación radiológica de que la fractura aún se encontraba en actividad. Se descartaban los casos en los que el dolor desaparecía y en los que existía compresiones superiores a la anteriormente referida.

Conforme se ha ido ganando en experiencia, se ha ampliado la horquilla terapéutica, alcanzándose en la actualidad las siguientes indicaciones ${ }^{79}$ :

1. Fracturas vertebrales de origen osteoporótico

2. Hemangiomas sintomáticos ${ }^{29,46}$

3. Metástasis óseas osteolíticas ${ }^{14,50,60,100}$

4. Mieloma múltiple $14,23,26,52,60,66$

5. Otras alteraciones:
a. Linfoma ${ }^{26}$
b. Espondilitis de Kummell ${ }^{22}$
c. Histiocitosis ${ }^{8}$
d. Osteogénesis imperfecta ${ }^{82}$

6. Nuevas tendencias:

a. Fracturas vertebrales traumáticas (casos seleccionados $)^{2}$

Podríamos hablar de contraindicaciones absolutas y relativas $^{9,65}$ :

\section{$\underline{\text { Absolutas }}$}

1. Infección localizada en la vértebra a tratar (osteomielitis, discitis, absceso)

2. Coagulopatía rebelde a tratamiento

3. Dolor no relacionado con el colapso vertebral

4. Fracturas antiguas asintomáticas

5. Tratamiento médico efectivo

$\underline{\text { Relativas }}$

1. Vértebra plana (cuando la perdida de altura del soma vertebral es superior a dos tercios de la altura previa)

2. Compresión neural por proceso neoformativo

3. Intrusión del muro posterior en el conducto vertebral

4. Alergia a alguna de las sustancias utilizadas en el procedimiento (cemento, contraste...)

Las limitaciones vendrían dadas porque el paciente no pueda tolerar una anestesia general o un decúbito prono prolongado.

\section{Semejanzas y diferencias entre ambos procedimientos}

Son procedimientos mínimamente invasivos, ideados para el tratamiento de las fracturas vertebrales secundarias a osteoporosis con el fin de aliviar el dolor, reforzar la columna y permitir una rápida movilización del paciente. La principal diferencia entre ambos procedimientos es que con el último se consigue restaurar en mayor grado la altura del cuerpo vertebral, buscando con ello reducir la deformidad espinal ${ }^{90}$.

\section{Estudio complementario preprocedimiento}

Una vez detectada una fractura, por lo general tras un

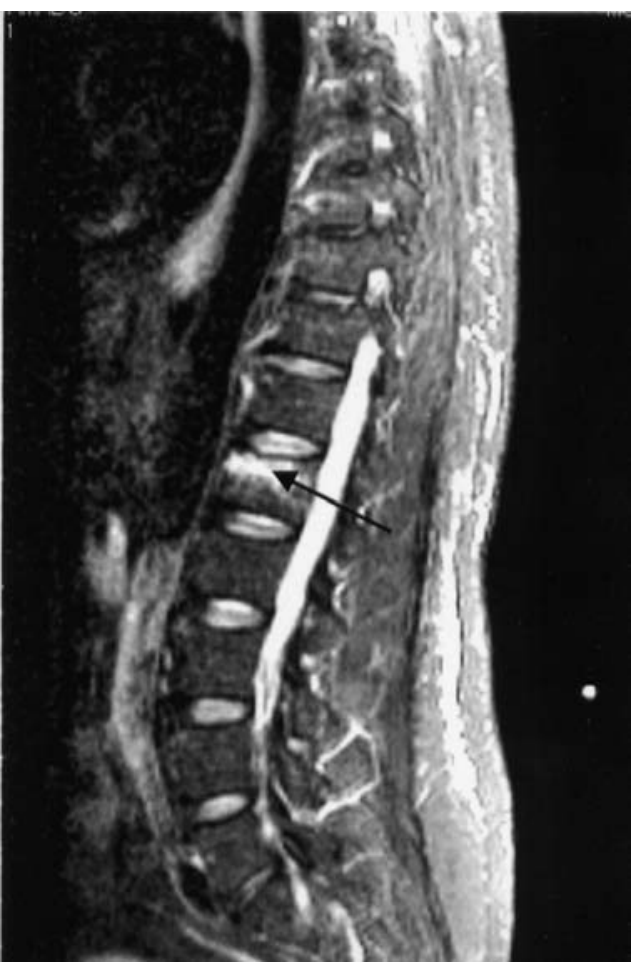

Figura 1. Resonancia magnética en secuencia STIR que muestra en el soma de LI una zona de hiperintensidad (flecha), correspondiente a una fractura en actividad.

estudio radiológico simple, el mejor método para conocer si la misma mantiene actividad es la resonancia magnética, sobre todo en la secuencia STIR (short tau inversion recovery) que muestra con claridad el edema que se aprecia en una fractura aguda por compresión. En esta secuencia el edema aparece hiperintenso ${ }^{3}$ (Figura 1). En aquellos pacientes con fracturas múltiples, debe definirse el nivel sintomático, aquel que mantenga actividad (edema en la resonancia $)^{20}$. Si el paciente es portador de marcapasos, puede resultar adecuado un rastreo óseo con tecnecio-99 $\left(\mathrm{Tc}^{99}\right)^{69}$. El escáner es útil en patología osteolítica, para demostrar la desestructuración de la vértebra, así como para valorar retropulsiones del muro posterior en el conducto vertebral ${ }^{9}$.

\section{Aspectos biomecánicos}

Una de las hipótesis que se barajan para justificar el efecto analgésico que se obtendría con ambos procedimientos se basa en el efecto mecánico que la cementación produciría al estabilizar el foco de fractura y eliminar los micro-movimientos que se originarían en el mismo ${ }^{79}$. Sin embargo, Erickson y colaboradores abogan más por la reacción exotérmica que acompaña a la polimerización del cemento como origen del alivio del dolor ${ }^{25}$.

Si se comparan los resultados biomecánicos, en cadá- 
ver, entre la vertebroplastia y la cifoplastia, no se encuentran diferencias ${ }^{102}$. Es más, se aprecia una resistencia mecánica similar con ambos procedimientos, aunque una mejor recuperación de la altura vertebral mediante la cifoplastia ${ }^{5}$. Belkoff et al. establecieron que para restaurar la resistencia a la fractura de una vértebra (en el cadáver), es suficiente con una pequeña cantidad de cemento: $4.4 \mathrm{ml}$ en la región lumbar, $3.1 \mathrm{ml}$ en la región toráco-lumbar, y $2.5 \mathrm{ml}$ en el resto de niveles torácicos ${ }^{5}$.

Por último, todavía no se han definido los posibles efectos adversos que el cemento puede inducir en las estructuras adyacentes ${ }^{94,101}$.

\section{Resultados y complicaciones}

Un sustancial alivio del dolor, (entre un 73-97\%), se ha comunicado en la mayoría de los pacientes tratados, tanto con vertebroplastia como con cifoplastia ${ }^{4,15,17,24,34,53,58,59,64,67,91,97}$ manteniéndose el resultado al año del procedimiento ${ }^{16,36,55,105}$.

Phillips et al. y Watts et al. han establecido recientemente la capacidad de la cifoplastia para recuperar la altura vertebral y mejorar el alineamiento espinal ${ }^{78,97}$. La recuperación media de la altura medida con radiografía lateral fue de $1.5 \mathrm{~mm}$ en la porción posterior del cuerpo vertebral, $4.7 \mathrm{~mm}$ en la porción media y $3.7 \mathrm{~mm}$ en la porción anterior ${ }^{51,91}$. Aunque no se puede establecer una regla, se consiguen mejores recuperaciones de la altura vertebral a mayor precocidad en el tratamiento ${ }^{17,38,42,59}$.

Por otro lado, se ha relacionado el alivio del dolor vertebral, obtenido con ambos procedimientos, con una reducción de la cifosis que sufren estos pacientes, al disminuir la contractura paravertebral y permitir una posición más erecta $^{90,91}$. A su vez esta corrección de la cifosis mejoraría la función pulmonar.

La proporción de complicaciones comunicadas con ambos procedimientos es escasa ${ }^{54,63,79,97}$. Sin embargo, la extravasación del cemento más allá de los confines de la vértebra resulta frecuente en la vertebroplastia, comunicán-

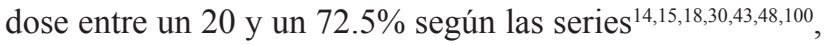
lo que podría acarrear un daño médulorradicular ${ }^{40,62,83}$ o un embolismo a distancia ${ }^{10,13,104}$, aunque, por lo general, no suelen tener repercusión clínica ${ }^{74,76}$. (Figura 2). En este sentido, Chiras apreció, como consecuencia de la fuga de cementos, radiculopatía en sólo el 5\% de su serie y compresión medular en menos del $0.5 \%{ }^{12}$, mientras Gangi et al. apreciaron radiculopatía en el $0,3 \%$ de la suya ${ }^{30}$. Con la cifoplastia se reducen las complicaciones por extravasación de cemento a niveles inferiores al $1 \%{ }^{80,94}$. En un registro multicéntrico de 1439 pacientes con 2194 fracturas tratadas mediante cifoplastia, el porcentaje de complicaciones graves alcanzó el $0.2 \%$ por fractura ${ }^{32}$.

El riesgo de las complicaciones aumenta con la

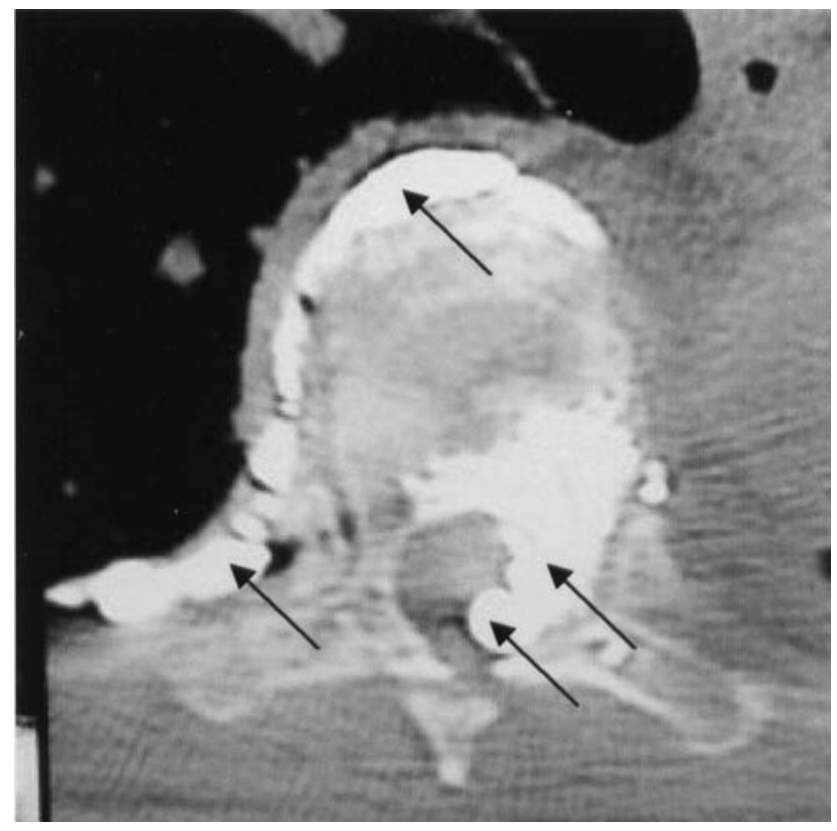

Figura 2. Vertebroplastia dorsal. Extravasación de cemento (flechas).

vascularización de las lesiones y con la desestructuración vertebral. Una forma de reducir el riesgo de fuga en la vertebroplastia sería conocer el drenaje venoso del cuerpo vertebral a reforzar (vertebrografía) antes del procedimiento $^{37}$. Mediante este estudio se podría prever hacia dónde migraría el cemento en caso de establecerse una fuga (hacia el conducto espinal -riesgo de compresión medular o estenosis-; hacia el foramen intervertebral riesgo de compresión radicular-; hacia la vena cava -riesgo de embolismo pulmonar-). En este sentido, McGraw et al. establecieron que la vertebrografía permite definir el flujo del cemento durante la vertebroplastia en el $83 \%$ de los $\operatorname{casos}^{70}$. Otros autores, sin embargo, no son partidarios de una vertebrografía previa, arguyendo que las densidades del contraste y del cemento son distintas, y que por lo tanto son sustancias que se comportan de forma diferente ${ }^{79}$, o que incluso el contraste podría quedar acantonado y crear una "mancha radioscópica" que dificultara la administración del cemento ${ }^{21}$. Otros autores concluyen que la vertebrografía no incrementa la seguridad del procedimiento ${ }^{35,96}$. A pesar de la controversia existente, y de que la vertebrografía no incremente la seguridad del procedimiento cuando se realiza por manos experimentadas, consideramos que puede ser una herramienta útil en manos poco versadas y ayudar a que la vertebroplastia se realice de forma más segura, buscando una rápida detención de la extravasación del cemento.

Para reducir aún más los riesgos, si cabe, estos procedimientos deben realizarse en un medio que disponga de una visualización radioscópica uni- (brazo en C) o 
bi-planar de alta calidad (que será continua y en proyección lateral mientras dure la administración de cemento, poniendo especial atención a la progresión del mismo hacia el muro posterior del cuerpo vertebral) y un equipo quirúrgico dispuesto a actuar en caso de complicaciones ${ }^{64}$. Hay autores que combinan la intensificación de la imagen con la tomografía computarizada ${ }^{31}$. Se deben evitar las complicaciones derivadas de una mala técnica en la colocación del instrumental ${ }^{94}$.

Una de las preocupaciones del refuerzo vertebral es que pueda aparecer una nueva fractura en un segmento adyacente al reforzado ${ }^{41}$. Parece ser que este fenómeno es más frecuente si la vértebra reforzada se encuentra próxima a la charnela toraco-lumbar y si además la recuperación de su altura es máxima ${ }^{49}$. Watts et al. han establecido que uno de cada 5 pacientes que se someten a una vertebroplastia puede desarrollar una segunda fractura ${ }^{97}$. Recientemente Uppin et al. han señalado que un $12 \%$ de los 177 pacientes que sometieron a una vertebroplastia desarrollaron una segunda fractura, dos tercios de las cuales ocurrieron en los primeros 30 días, y el 66\% de las mismas en una vértebra adyacente ${ }^{95}$. Otros aprecian una mayor incidencia de fracturas secundarias en los dos primeros meses tras el procedimiento $^{27}$. Lin et al. han encontrado una correlación entre la existencia de una fuga intradiscal de cemento con una nueva fractura en el soma de la vértebra adyacente ${ }^{61}$. Por otro lado, se debe tener en cuenta, que en la historia natural de la osteoporosis espinal, una vez que se alcanza el umbral de fractura, el desarrollo de múltiples fracturas es lo habitual ${ }^{9}$.

\section{Coste}

El coste medio del Kit de vertebroplastia (sin cemento) en EEUU es de 400 dólares, mientras que el kit de cifoplastia (sin cemento) alcanza los 3400 dólares ${ }^{68}$. En España, el coste con cemento incluido es de aproximadamente 1100 euros y 4250 euros respectivamente (datos ofrecidos por el distribuidor).

\section{Técnica}

\section{Aspectos generales}

\section{Cuidados preoperatorios y consentimiento informado}

Como ante cualquier intervención quirúrgica, es necesario la realización de un estudio preoperatorio, que incluya como mínimo una bioquímica sanguínea, una hematimetría, un recuento y fórmula leucocitaria y un estudio de coagulación.

Se deberá explicar de forma detallada lo que se va a realizar al paciente, los pros, contras y alternativas, así como los posibles riesgos, debiendo el paciente firmar el

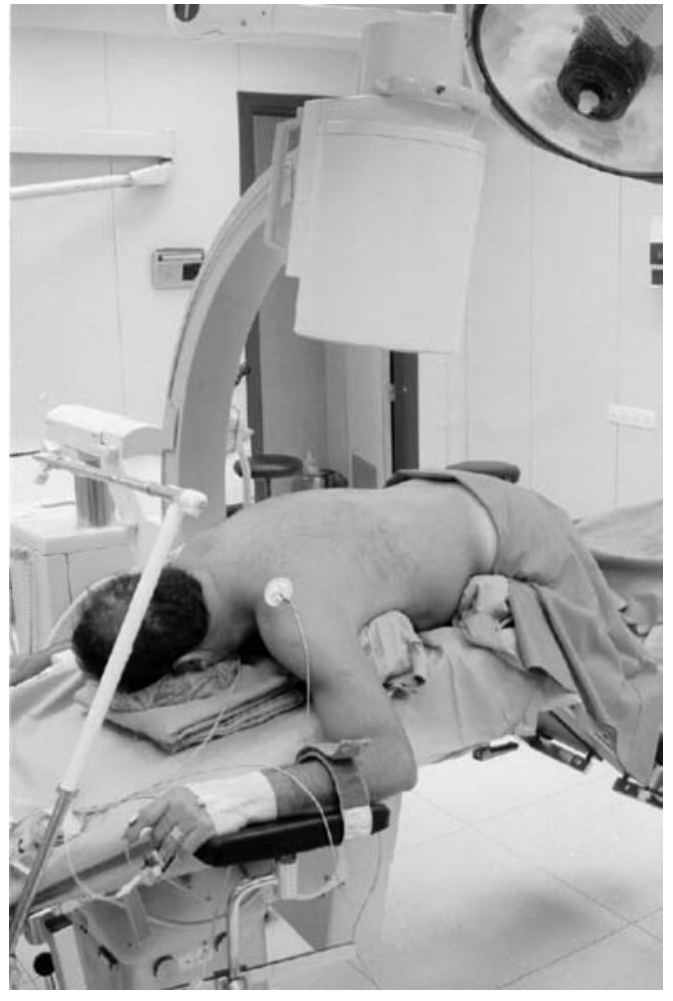

Figura 3. Colocación del paciente para una cifoplastia dorsal; decúbito prono.

consentimiento informado.

De forma habitual administramos por vía parenteral, como medicación preoperatoria, un gramo de cefazolina una hora antes de la intervención.

\section{Anestesia}

Ambos procedimientos pueden realizarse con una sedación consciente o bajo anestesia general. Por lo general se prefiere la sedación consciente para la vertebroplastia, puesto que el paciente puede ser interrogado en cualquier momento del procedimiento, o puede comunicar cualquier sensación extraña que experimente.

\section{Colocación del paciente}

Puede optarse por un decúbito lateral o prono sobre una mesa radiotransparente, según la situación clínica del paciente o el procedimiento a realizar. Preferimos el decúbito prono en caso de llevar a cabo una cifoplastia o cuando haya que reforzar niveles torácicos. Siempre habrá que prestar atención al almohadillado de las zonas de apoyo (Figura 3).

\section{Características del cemento}

En la actualidad se utiliza un cemento de alta viscosidad y radioopaco, resultado de la combinación de un polvo compuesto por un copolirriero del metil-metacrilato, sulfato 
de bario y peróxido de benzoilo, con un líquido compuesto fundamentalmente por un monómero de metil-metacrilato. $\mathrm{Al}$ polimerizarse, el cemento se endurece provocando una reacción exotérmica. Deramond et al. apreciaron, in vitro, que esta elevación térmica no produce daño neurológico ${ }^{19}$.

Debe tenerse en cuenta, que el componente líquido es un potente solvente de lípidos y que puede causar dermatitis por contacto.

Se puede retrasar el tiempo de polimerización del cemento enfriando sus componentes antes de su uso y manteniendo el cemento en un baño helado ${ }^{11}$.

Recientemente distintos grupos de trabajo han analizado las características de distintos cementos y sus aspectos biomecánicos para su uso con técnicas de refuerzo vertebral $^{47,81,93}$.

\section{Cuidados postoperatorios}

- Reposo relativo en cama entre 6 y 12 horas, iniciándose tras este tiempo primero la sedestación y posteriormente la deambulación.

- Control de constantes habituales

- Analgesia estándar

- TAC de control. A partir de las primeras 24 horas. Importante para establecer la extensión del relleno vertebral y para excluir la extravasación del cemento.

- Si no hay incidencias, alta hospitalaria a partir de las primeras 24 horas.

- Corsé para evitar la flexión del tronco durante un mes.

Kits de cada procedimiento.

\section{Kit para una vertebroplastia}

Trocar de punción de $30 \mathrm{~cm}$ de longitud y de $17 \mathrm{G}$ de calibre (abordaje posterolateral)

Trocar de punción de $16 \mathrm{~cm}$ de longitud y de $17 \mathrm{G}$ (abordaje transpedicular)

Aguja-cánula de $16 \mathrm{~cm}$ de longitud y de 14 G Sistema de inyección controlada

\section{Kit para una cifoplastia} bre

Trocar de punción: aguja de Jamshidi de 11 G de cali-

Alambres guía (romo o con punta)

Introductor óseo

Cánula de trabajo de $4.2 \mathrm{~mm}$ de diámetro

Broca manual de precisión

Cateter de balones inflables, uni o bidireccionales, de 10,15 o $20 \mathrm{~mm}$

Jeringa de inflado controlado

Introductores del cemento óseo (bone filler) (cánula -de menor luz que la de trabajo- y estilete)

Enhebrado percutáneo del pedículo para un abordaje transpedicular estándar (bajo control radioscópico). Este aspecto es común a las dos técnicas. Pasos:

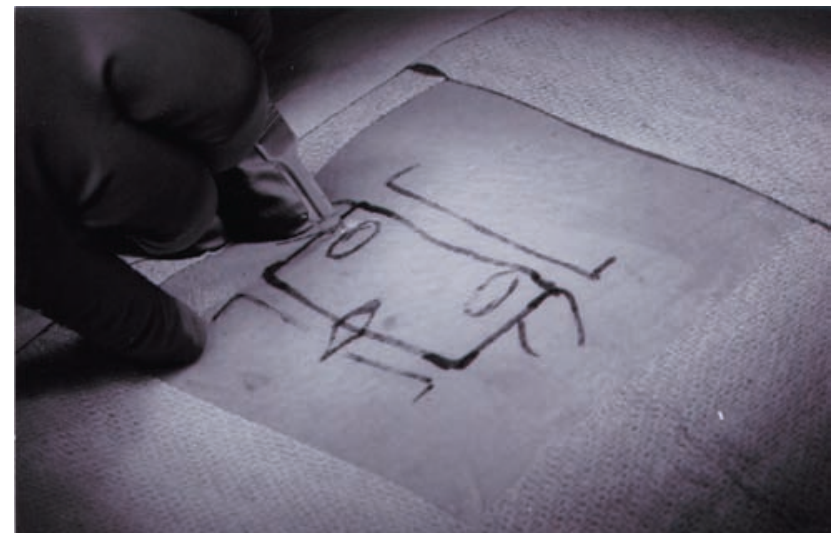

Figura 4. Se muestra la silueta de la vértebra dibujada sobre la piel. Abordaje a $1 \mathrm{~cm}$ por fuera del pedículo izquierdo.

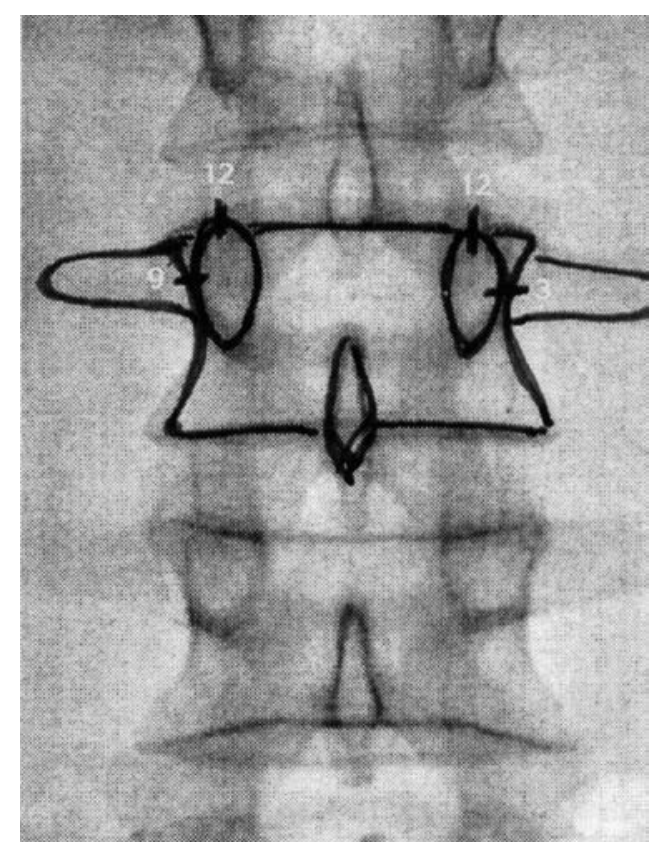

Figura 5. Imagen que debe buscarse por radioscopia. Obsérvese la disposición de los pedículos.

1. Localización pedicular por radioscopia. Imprescindible: se deberá abortar el procedimiento si no se aprecia.

2. Antes de incidir la piel, localizar el pedículo sobre la misma. Para ello puede utilizarse cualquier elemento radioopaco. Puede resultar útil dibujar la silueta de la vértebra en la piel.

3. Hacer una incisión en la piel de 2-3 mm: $1 \mathrm{~cm}$ por fuera del punto de entrada hacia el pedículo $(2 \mathrm{~cm}$ por fuera en el nivel L5) (Figura 4).

4. Colocación inicial del punzón iniciador: Este paso debe iniciarse en proyección A-P (Figura 5).

a. En proyección A-P. Si imaginamos el pedículo como la esfera de un reloj, la entrada la haremos 


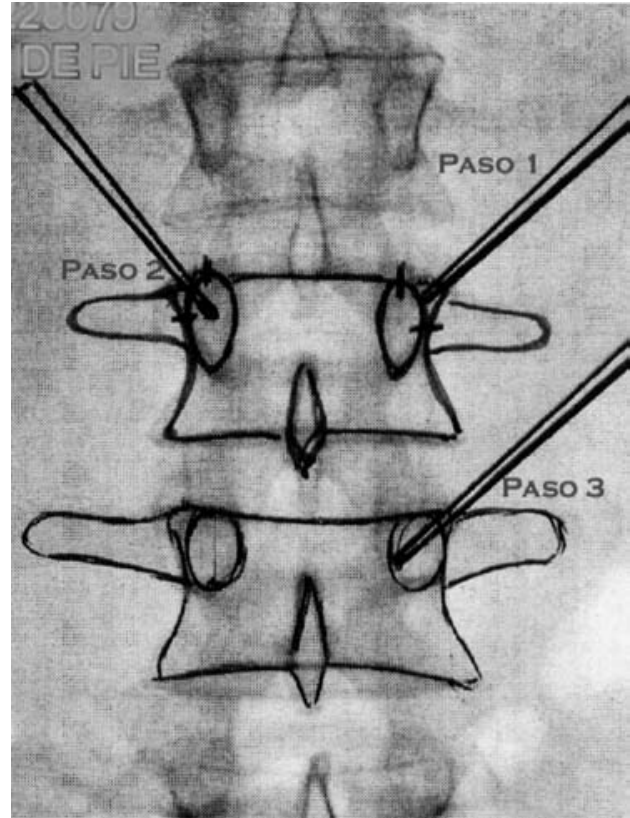

Figura 6. Pasos sucesivos para el enhebrado pedicular en proyección AP.

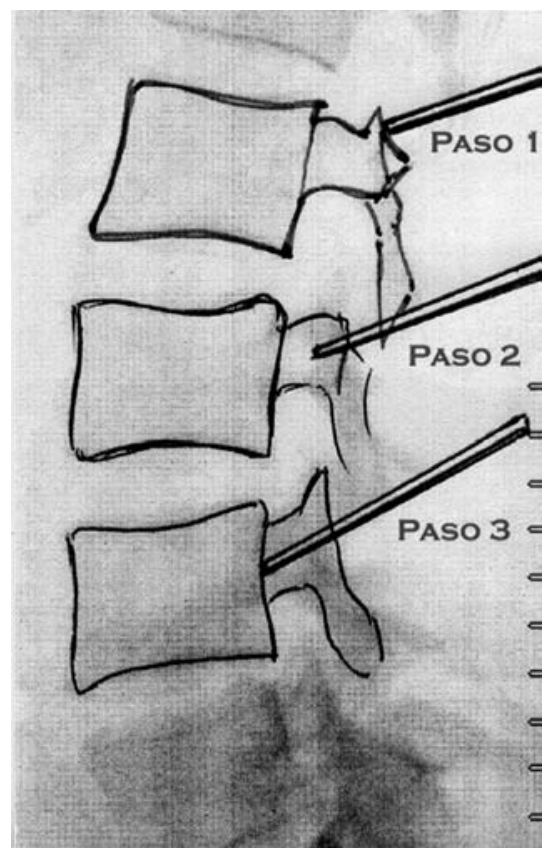

Figura 7. Pasos sucesivos para el enhebrado pedicular en proyección lateral.

entre las 11 y las 9 para el pedículo izquierdo, ó entre la 1 y las 3 para el pedículo derecho.

b. En proyección lateral. Verificar que la proyección de la trayectoria del trocar dentro del cuerpo vertebral es la adecuada para asegurar una reducción de la fractura. Se deben comprobar los márgenes superior e inferior del pedículo

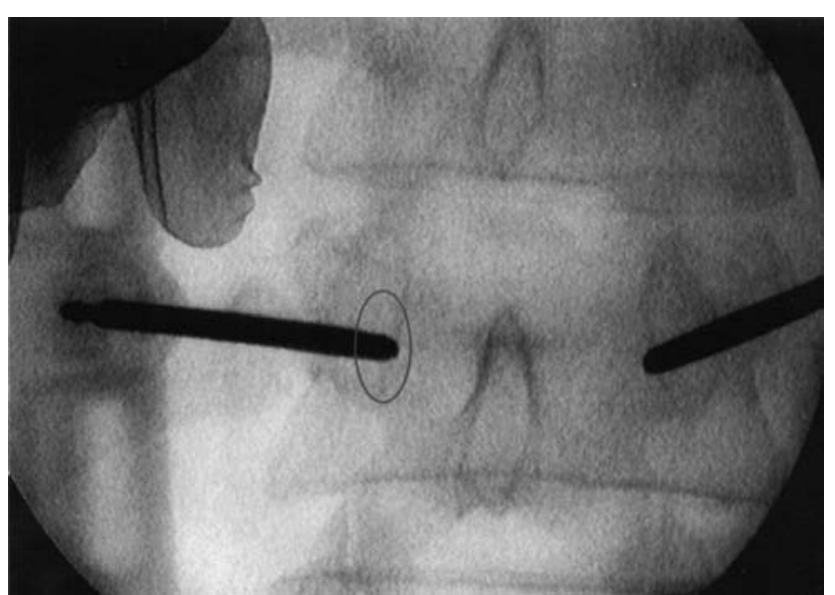

Figura 8. Imagen radioscópica en proyección A-P. Ubicación de los trócares.

5. Introducción progresiva del punzón iniciador hasta alcanzar el muro posterior del cuerpo vertebral (Figuras 6 y 7). (ADVERTENCIA: este avance deberá realizarse en proyección lateral, pudiéndose comprobar la situación de la punta del trocar iniciador en proyección A-P en cualquier momento, sabiendo que siempre deberá encontrarse la misma por dentro de los límites del pedículo). Al alcanzar el muro posterior comprobaremos que:

a. En proyección lateral: la punta del trocar quedará tocando el muro posterior del cuerpo vertebral

b. En proyección A-P: A nivel del muro posterior del cuerpo vertebral, la punta del trocar deberá permanecer por dentro del margen interno del pedículo. Se deberá prestar atención a este detalle, puesto que, si se ha sobrepasado el margen interno del pedículo, la punta del trocar se encuentra por dentro del mismo y por lo tanto medial, con lo que existe riesgo de lesionar estructuras neurológicas.

6. Una vez que se alcanza el muro posterior, y se han hecho las comprobaciones en A-P y lateral se procede a introducir el trocar $2 \mathrm{~mm}$ dentro del cuerpo vertebral (Figura 8).

7. A partir de aquí existen peculiaridades para cada procedimiento.

\section{Vertebroplastia}

Se han descrito diferentes vías de abordaje que podrán utilizarse según las características de cada caso ${ }^{64}$. De forma habitual se utiliza el abordaje posterolateral para el nivel lumbar, el abordaje transpedicular (o intercostovertebral) para el nivel dorsal y el anterolateral (o transoral) para el cervical ${ }^{44}$. Una vez alcanzado con el trocar de trabajo el soma vertebral, éste se avanzará (bajo control radiológico en proyección lateral) hasta alcanzar la unión de los ter- 
cios medio y anterior ( $\sin$ sobrepasar en proyección AP la apófisis espinosa). Una vez situado, podrá realizarse una vertebrografía (opcional) para conocer la distribución del flujo vascular vertebral (conforme se consiga experiencia este paso podría obviarse). Tras ella, se procederá a la inyección del cemento, que en general se aplica bajo condiciones de "presión elevada", por lo que su administración debe realizarse lentamente, bajo control radioscópico continuo. Esta inyección deberá interrumpirse si se observan fugas o cuando se produzca una elevada resistencia a su administración. Parece ser que se registra una mayor presión de administración cuanto mayor sea el calibre de la cánula con la que se aplica el cemento y cuanta mayor proporción de cemento se inyecte ${ }^{1}$. No existe una cantidad mínima de cemento a inyectar para que el procedimiento sea efectivo ${ }^{4}$. Por regla general se administra entre 2 y 11 $\mathrm{ml}$ por vértebra ${ }^{48}$. Los pormenores del procedimiento han sido publicados con anterioridad ${ }^{64}$.

\section{Cifoplastia}

Debemos establecer la vía de abordaje con la que proceder. Por lo general se utiliza el abordaje transpedicular entre los niveles décimo torácico y quinto lumbar $\left(\mathrm{D}_{10}-\mathrm{L}_{5}\right)$, mientras que se podrá usar un abordaje extrapedicular entre los niveles torácicos quinto y decimosegundo $\left(\mathrm{D}_{5}-\mathrm{D}_{12}\right)$.

Una vez alcanzado con el trocar de punción el soma vertebral, se retira el estilete del mismo, y en proyección lateral, se introduce un alambre guía a su través hasta aproximadamente la mitad del cuerpo vertebral. Una vez posicionado, se retira el trocar y utilizamos el alambre guía para ubicar el introductor óseo. Posteriormente disponemos la cánula de trabajo usando el introductor óseo de guía, hasta 1-2 mm más allá del muro posterior del cuerpo vertebral. Se retira el introductor óseo y se deja posicionada la cánula de trabajo (Figura 9).

En este momento podemos realizar una toma biópsica si fuera necesario.

A continuación, y todavía en proyección lateral, se introduce la broca manual de precisión para labrar un canal en el soma vertebral. Cuando en la proyección lateral la broca alcance la mitad del cuerpo vertebral, se debe comprobar la posición de la misma en la proyección A-P, que se deberá mostrar a medio camino entre el margen interno del pedículo y la apófisis espinosa (Figura 10). Se debe dejar de perforar con la broca manual a 2-3 mm del córtex anterior. Al alcanzar el punto final de perforación, la punta de la broca debe parecer que toca la apófisis espinosa, en proyección A-P. Tras retirar la broca, se puede introducir en el canal perforado, el sistema de relleno óseo (con su estilete) a modo de baqueta, para facilitar el alojamiento del catéter de balón inflable, y evitar que alguna espícula ósea pueda dañar el balón.

Tras esto, insertaremos dicho catéter hasta unos $4 \mathrm{~mm}$
$2005 ; 16: 427-440$

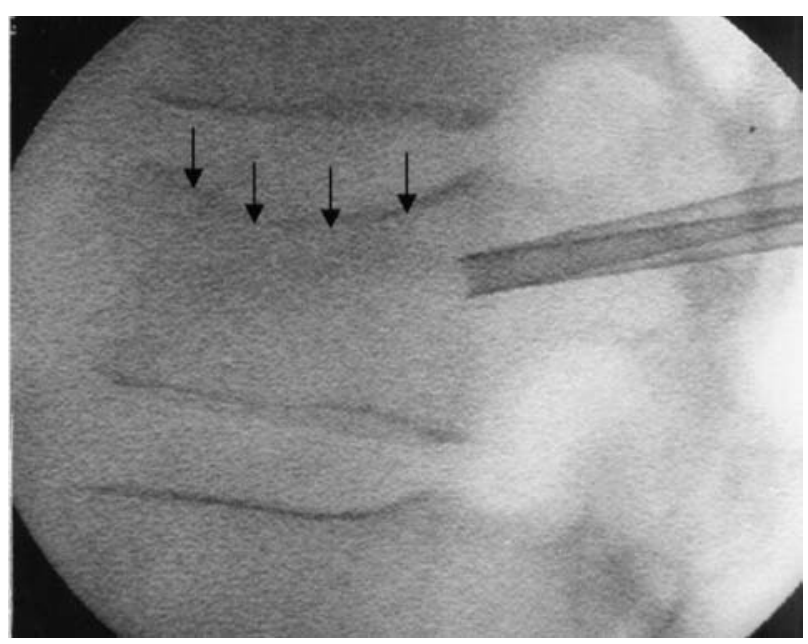

Figura 9. Imagen de radioscopia en proyección lateral. Posicionamiento de las cánulas de trabajo. Fractura de platillo superior (flechas).

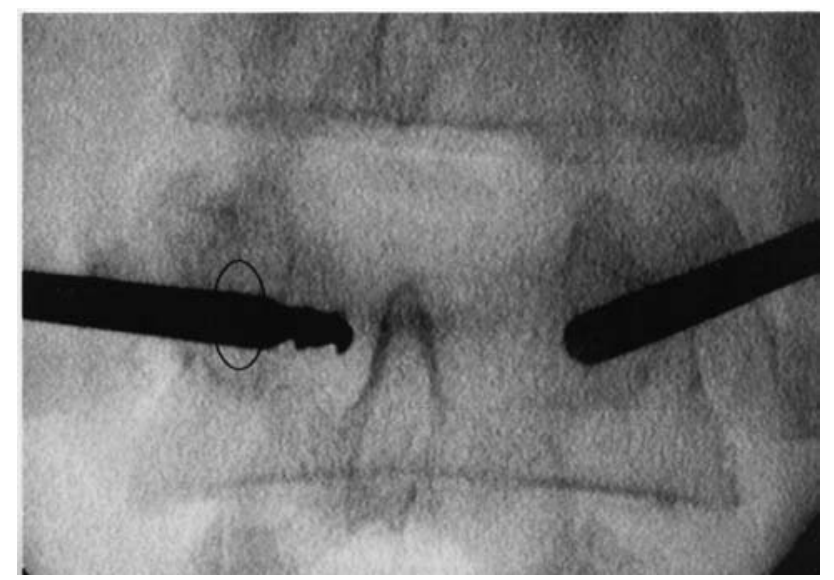

Figura 10. Imagen radioscópica en proyección $A-P$. Pediculo izquierdo: Broca de precisión próxima al final de su recorrido. Pedículo derecho: cánula de trabajo.

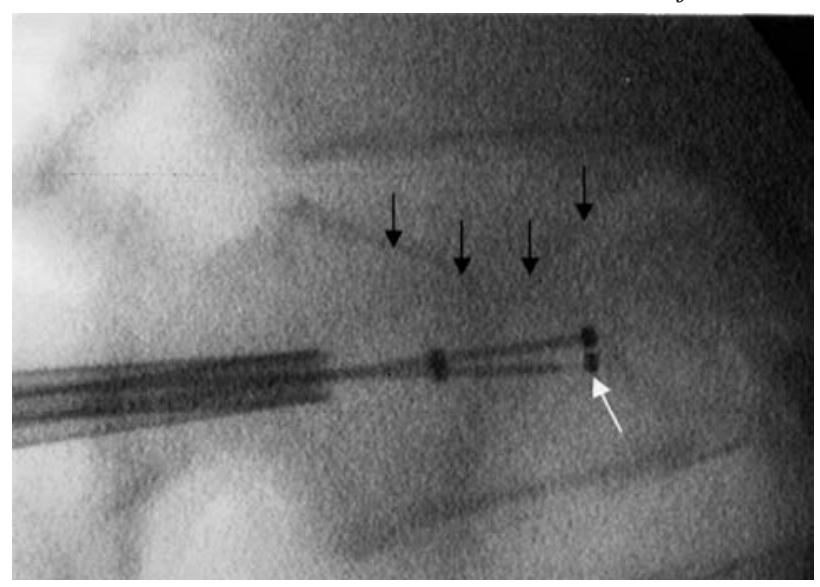

Figura 11. Imagen de radioscopia en proyección lateral. Alojamiento de los catéteres de balón. Se puede reconocer los testigos (flecha blanca). Fractura de platillo superior (flechas negras). 
del córtex anterior del cuerpo vertebral (figura 11). Lo insuflaremos hasta un nivel de 50 psi (-libras por pulgada cuadrada- ó 3,4 atmósferas) para asegurar su posición (que no se desplace) mientras se procede a repetir los pasos en el pedículo opuesto. Una vez ubicado el balón contralateral, se insuflarán ambos balones con incrementos progresivos de 0.5 cc. Apreciaremos una mayor presión de inflado del balón, cuanto mayor sea la densidad ósea y cuanto más tiempo haya pasado de la instauración de la fractura.

Se debe controlar por radioscopia la posición de los balones en relación con las corticales del cuerpo vertebral (Figuras 12, 13 y 14). El límite de inflado de los balones es:

a. Cuando se realinien los platillos vertebrales

b. Cuando se alcance la presión máxima que admite el balón (si se sobrepasa existirá riesgo de rotura del mismo)

c. Cuando se consiga el vólumen máximo del balón (si se sobrepasa existirá riesgo de rotura del mismo)

d. Cuando se alcance la cortical de cualquier pared

Alcanzado el límite de inflado, se procederá a la preparación del cemento óseo radioopaco. Una vez listo y en estado de baja viscosidad, se carga en los utensilios dispuestos a tal efecto (bone filler) y se espera hasta que el cemento se torne viscoso. Tras retirar los balones (podría apreciarse entonces una zona de radiotransparencia en la imagen de radioscopia), se alojan los sistemas de relleno a 3-5 mm del córtex anterior del cuerpo vertebral, y bajo radioscopia continua, se inyecta el cemento lentamente mediante un estilete que al introducirse a su vez en el sistema de relleno, empuja el cemento hacia la cavidad creada. Durante esta fase se debe mantener el sistema de relleno anterior. Cada bone filler alberga como máximo 1,5 cc de cemento. Inyectaremos cemento hasta:

I. Que el cemento se mantenga $2 \mathrm{~mm}$ por dentro del muro posterior

II. Extravasación

III. Relleno vertebral adecuado

El procedimiento de cementación se repetirá en el lado contralateral.

Una vez que el relleno se ha completado, se retiran las cánulas y se cierran las incisiones (Figuras 15 y 16).

En ocasiones se debe utilizar un abordaje extrapedicular (para los niveles $\mathrm{D}_{5}-\mathrm{D}_{12}$ ). En este caso se deberá tener en cuenta lo siguiente:

- En proyección A-P, los pedículos aparecerán más altos en el cuerpo vertebral.

- El punto de entrada será más alto, por fuera de los pedículos, entre la costilla y la apófisis transversa por encima y lateral a la pared del pedículo, para permitir un adecuado posicionamiento del instrumental(superoinferior).

- Se deberá verificar el punto de entrada en proyección

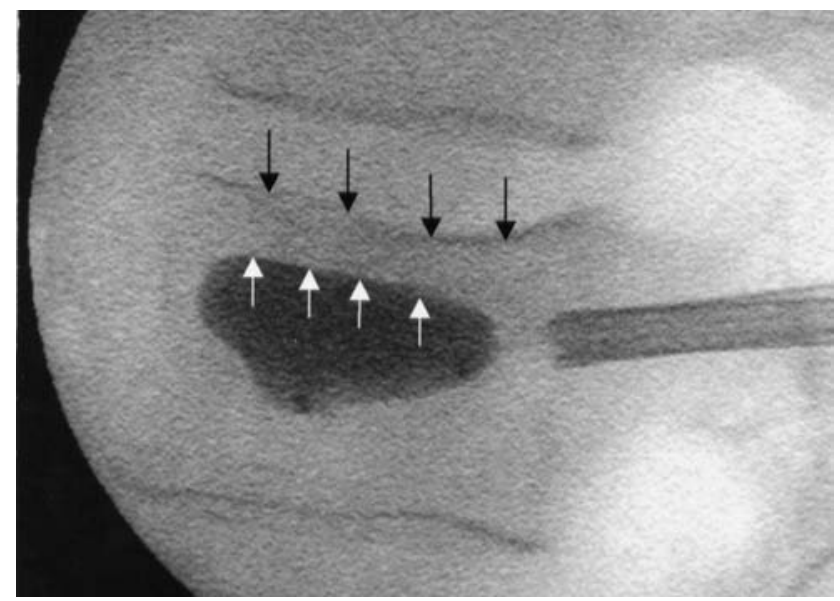

Figura 12. Imagen de radioscopia en proyección lateral. Insuflado progresivo de los balones (flechas blancas) hasta conseguir la recuperación de la fractura de platillo superior (flechas negras).

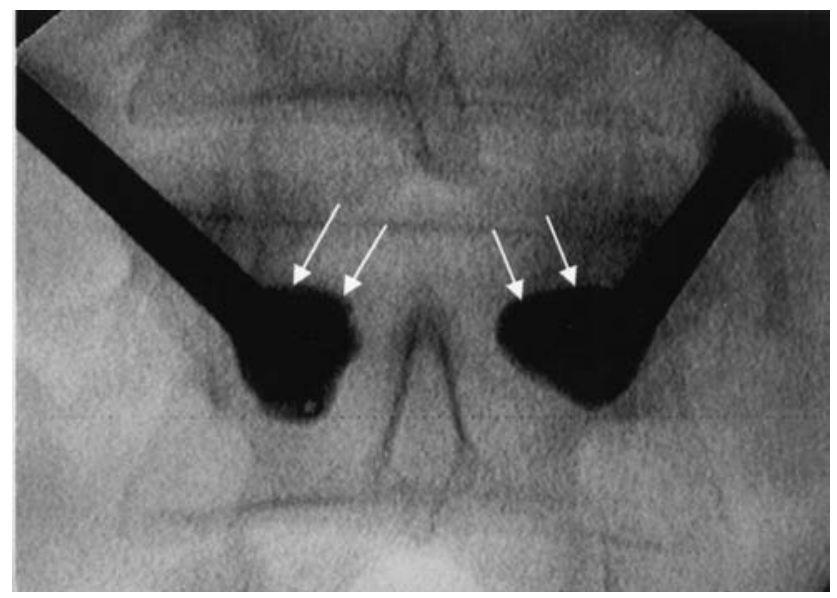

Figura 13. Imagen de radioscopia en proyección A-P. Recuperación de la fractura de platillo superior. Mismo caso que el de la figura 11.

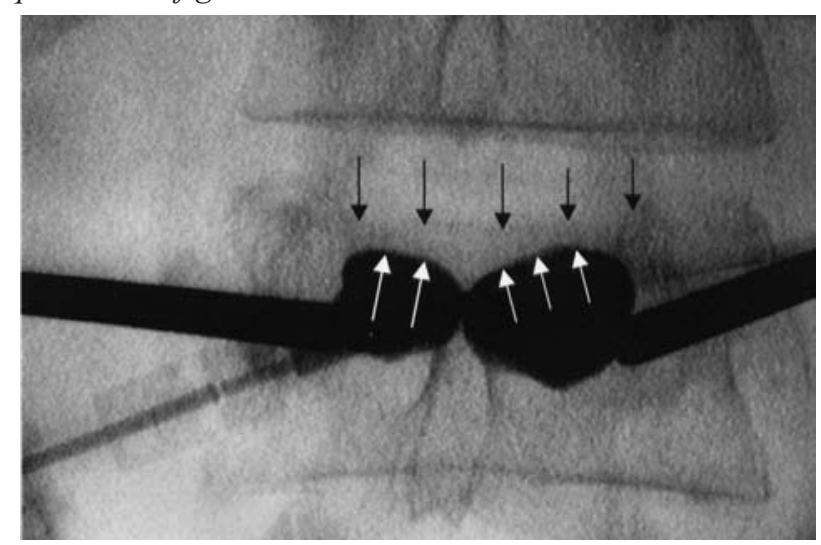

Figura 14. Imagen de radioscopia en proyección A-P. Insuflado progresivo de los balones (flechas blancas) hasta conseguir la recuperación de la fractura de platillo superior (flechas negras). 


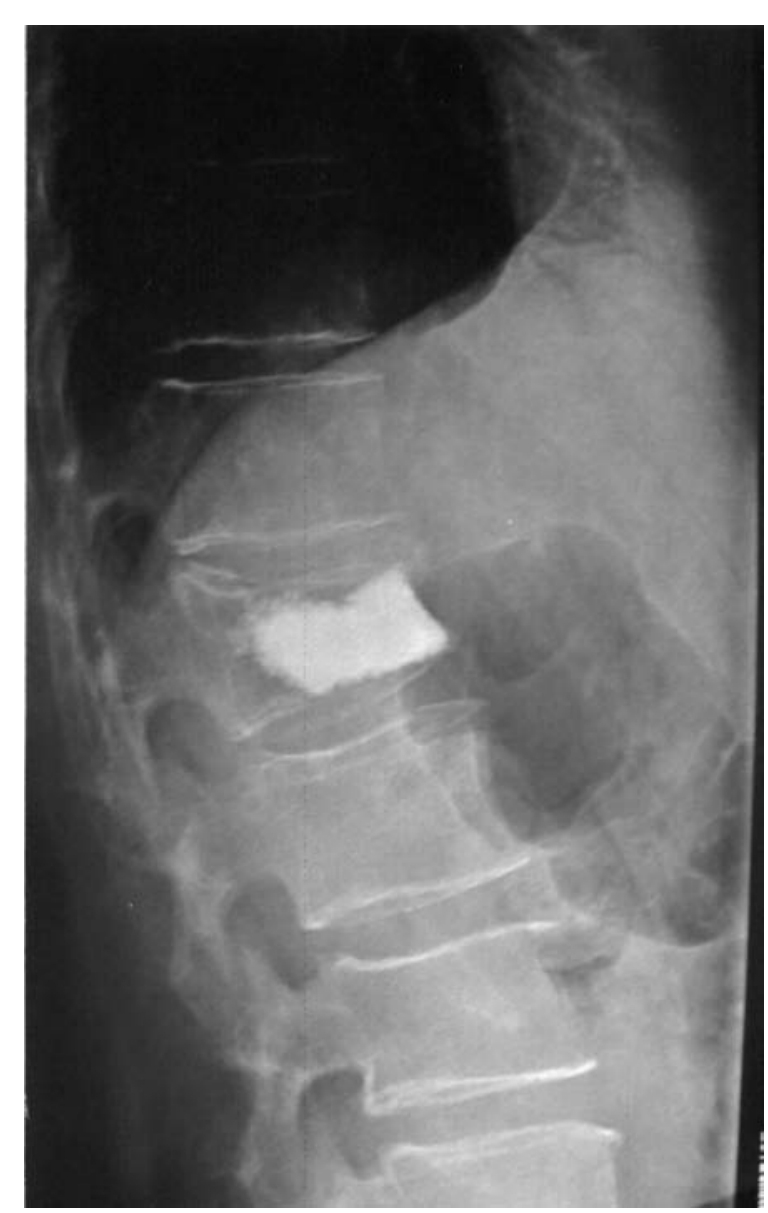

Figura 15. Control radiológico simple en proyección lateral. Cifoplastia de $\mathrm{Ll}$.

A-P con la proyección lateral.

Tras la inserción del trocar en el soma vertebral, la secuencia en el uso del instrumental y los controles de imágenes son idénticos al abordaje transpedicular.

\section{Dosis de radiación}

Entendemos por dosis absorbida, la dosis de radiación absorbida por el cuerpo. Se mide en GRAY (Gy). Un Gray se define a su vez como la energía registrada de un julio por kilogramo de materia. La evaluación del riesgo asociado a esta dosis absorbida se mide en SIEVERT (Sv). Un Sievert es equivalente a un gray multiplicado por un factor de ponderación consustancial a cada radiación y órgano.

Recientes estudios han demostrado una dosis de radiación excesiva en las manos del cirujano (0.5-8.5 mGy), al compararlas con las zonas protegidas por el delantal de plomo (0.01-0.47 mGy) $)^{71}$. Una exposición radioscópica de aproximadamente 10 minutos induce una dosis media en la piel del paciente de 173-233 mGy (para que se produzca un daño no estocástico en la piel -eritema- son precisas dosis
2005; 16: 427-440

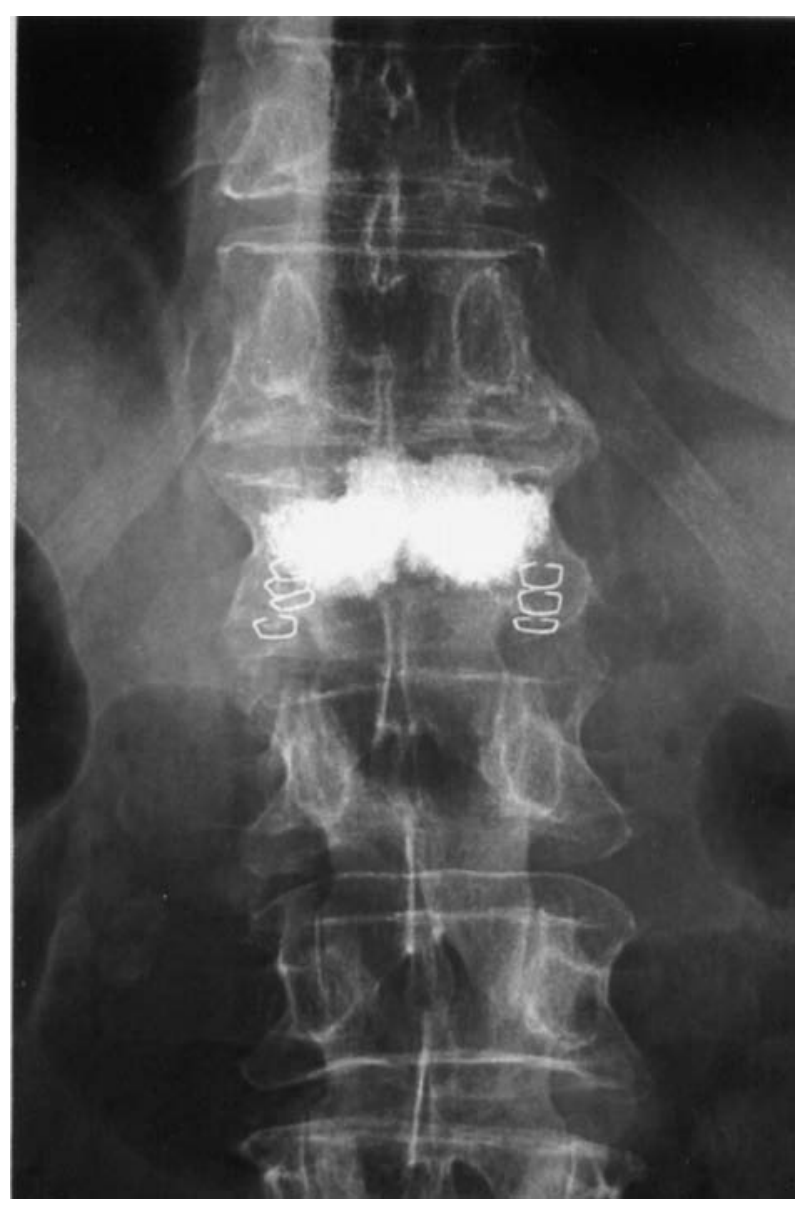

Figura 16. Control radiológico simple en proyección A-P. Cifoplastia de L1.

de 2 Gy) ${ }^{75,86}$.

Procedimiento quirúrgico a cielo abierto

Fue comunicado por primera vez por Wenger y Markwalder en $1999^{99}$. Este procedimiento permitiría además de realizar una determinada técnica quirúrgica -descompresión, fijación- llevar a cabo una técnica de refuerzo vertebral pudiendo controlar in situ una posible fuga de cemento óseo. Poco a poco este proceder se va extendiendo ${ }^{7,28}$.

\section{Conclusiones}

De lo referido hasta aquí, consideramos que las técnicas de refuerzo vertebral deben tenerse en cuenta y formar parte del arsenal terapéutico neuroquirúrgico por el beneficio que producen y por la escasa comorbilidad que provocan.

¿Cuándo deberíamos plantearnos el uso de una técnica de refuerzo vertebral?

En aquellas fracturas vertebrales secundarias a osteoporosis, que se mantengan activas, en las que persistan los 
síntomas a pesar de un tratamiento médico adecuado.

Como procedimiento coadyuvante en lesiones vertebrales neoformativas. Es importante reseñar que las técnicas de refuerzo vertebral no son procedimientos excluyentes, pudiéndose asociar, de ser necesario, un tratamiento radioterápico, quimioterápico o quirúrgico convencional ${ }^{9}$.

¿Qué procedimiento utilizar?

Debido a la diferencia en coste entre ambos procedimientos por un lado, y que a mayor antigüedad de la fractura existe una mayor dificultad para conseguir una corrección de la deformidad, por instauración de los fenómenos de consolidación, deberíamos plantearnos de entrada una vertebroplastia ante: fracturas en compresión de más de 6 meses de evolución, sintomática, y con una pérdida de altura vertebral de más del 50\%; fracturas en compresión con escasa deformidad, y hemangiomas vertebrales sintomáticos.

La cifoplastia se plantearía, de entrada, en las fracturas osteoporóticas en progresión, de reciente instauración, que además asocien una pérdida del alineamiento sagital o un incremento de la angulación segmentaria, en las que la recuperación de la altura vertebral es lo prioritario. Por otro lado, esta técnica resulta útil en las lesiones tumorales, ya que, al crear una cavidad en una vértebra tumoral, se evitaría el riesgo de fugas de cemento que con gran frecuencia acompaña a las vértebras desestructuradas que se refuerzan.

Aún no se dispone de datos de estudios prospectivos, aleatorizados que comparen ambos procedimientos o alguno de ellos con un tratamiento alternativo.

¿Profilaxis?

En este sentido se ha llevado a cabo un interesante estudio en cadáver por Higgins et al, en el que concluyen que la región torácica muestra un escaso beneficio con el refuerzo profiláctico ${ }^{41}$. Nos enfrentaríamos a discusiones éticas.

¿Hasta dónde son útiles estos procedimientos?

Con independencia de la técnica utilizada, la enfermedad ósea de base debe tenerse en cuenta, ya que estos procedimientos no evitan sus consecuencias. Deben buscarse los factores desencadenantes de dichas fracturas e intentar corregirlos, administrar tratamientos que incrementen la masa ósea, insistir en una actividad física adecuada y poner todos los medios posibles para evitar caídas.

Por otro lado, todavía no se ha resuelto el problema de la posible fractura del segmento adyacente.

\section{Bibliografía}

1. Agris, J.M., Zoarski, G.H., Stallmeyer, M.J.B., Ortiz, O.: Intervertebral pressure during vertebroplasty: a study comparing multiple delivery systems. Presented at the annual meeting of the American Society of Spine Radiology, Scottsdale, AZ, 2003; pp:19-23.
2. Arregui, R., Martínez Quiñones, J.V., Aso, J.: Fracturas dorsolumbares por flexo-compresión. Nuevas estrategias terapéuticas. IV Congreso de la Sociedad Española de Neurorraquis. Tenerife. 17-19 Febrero, 2004.

3. Baker, L.L., Goodman, S.B., Perkash, I., Lane, B., Enzmann, D.R.: Benign versus pathologic compression fractures of vertebral bodies: assessment with conventional spin-echo, chemical-shift, and STIR MR imaging. Radiology. 1990;174: 495-502.

4. Barr, I.D., Barr, M.S., Lemley, T.I., McCann, R.M.: Percutaneous vertebroplasty for pain relief and spinal stabilization. Spine. 2000; 25: 923-928.

5. Belkoff, S.M., Mathis, J.M., Fenton, D.C., et al.: An ex vivo biomechanical evaluation of an inflatable bone tamp used in the treatment of compression fracture. Spine 2001; 26: 151156.

6. Berlemann, U., Franz, T., Orler, R., Heini, P.F.: For treatment of osteoporotic vertebral fractures: a prospective non-randomized study. Eur Spine J. 2004; 13: 496-501.

7. Boszczyk, B.M., Bierschneider, M., Schmid, K., et al.: Microsurgical interlaminary vertebro - and kyphoplasty for severe osteoporotic fractures. J Neurosurg Spine. 2004; 100: 32-37.

8. Cardon, T., Hachulla, E., Flipo, R.M., et al.: Percutaneous vertebroplasty with acrylic cement in the treatment of a Langerhans cell vertebral histiocytosis. Clin Rheumatol. 1994; 13: 518-521.

9. Carrino, J.A., Chan, R., Vaccaro, A.R.: Vertebral augmentation: vertebroplasty and kyphoplasty. Semin Roentgenol. 2004; 39: 68-84.

10. Charvet, A., Metellus, P., Bruder, N., et al.: Pulmonary embolism of cement during vertebroplasty. Ann Fr Anesth Reanim. 2004; 23: 827-830.

11. Chavali, R., Resijek, R., Knight, S.K., Choi, I.S.: Extending polymerization time of polymethylmethacrylate cement in percutaneous vertebroplasty with ice bath cooling. AJNR Am J Neuroradiol. 2003; 24: 545-546.

12. Chiras, J.: Percutaneous vertebral surgery: techniques and indications. J Neuroradiol 1997; 24: 45-52.

13. Choe du, H., Marom, E.M., Ahrar, K., et al.: Pulmonary embolism of polymethyl methacrylate during percutaneous vertebroplasty and kyphoplasty. Am J Roentgenol. 2004; 183: 1097-1102

14. Cotten, A., Dewatre, F., Cortet, B., et al.: Percutaneous vertebroplasty for osteolytic metastases and myeloma: effects of the percentage of lesion filling and the leakage of methyl methacrylate at clinical follow-up. Radiology 1996; 200: 525530 .

15. Cortet, B., Cotten, A., Boutry, N., et al.: Percutaneous vertebroplasty in the treatment of osteoporotic vertebral compression fractures: an open prospective study. J Rheumatol. 1999; 26: 2222-2228.

16. Coumans, J.V., Reinhardt, M.K., Lieberman, I.H.: 
Kyphoplasty for vertebral compression fractures: 1-year clinical outcomes from a prospective study. J Neurosurg Spine. 2003; 99: 44-50.

17. Crandall, D., Slaughter, D., Hankins, P.J., et al.: Acute versus chronic vertebral compression fractures treated with kyphoplasty: early results. Spine J. 2004; 4: 418-424.

18. Deramond, H.: Percutaneous vertebroplasty with polymethylmethacrylate: technique, indication and results. Radiol Clin North Am 1988; 36: 533-546.

19. Deramond, H., Wright, N.T., Belkoff, S.M.: Temperature elevation caused by bone cement polymerization during vertebroplasty. Bone, 1999; 25 (2 Suppl): 17S-21S.

20. Do, H.M.: Magnetic resonance imaging in the evaluation of patients for percutaneous vertebroplasty. Top Magn Reson Imaging. 2000; 11: 235-244.

21. Do, H.M.: Intraosseous venography during percutaneous vertebroplasty: is it needed? Am J Neuroradiol. 2002; 23: 508-509

22. Do, H.M., Jensen, M.E., Marx, W.F., Kallmes, D.F.: Percutaneous vertebroplasty in vertebral osteonecrosis (Kummell's spondylitis). Neurosurg Focus (serial online). July 1999; article 2.

23. Dudeney, S., Lieberman, I.H., Reinhardt, M.K., Hussein, M.: Kyphoplasty in the treatment of osteolytic vertebral compression fractures as a result of multiple myeloma. J Clin Oncol. 2002; 20: 2382-2387.

24. Eck, J.C., Hodges, S.D., Humphreys, S.C.: Vertebroplasty: a new treatment strategy for osteoporotic compression fractures. Am 3 Orthop. 2002; 31:1 23-127.

25. Eriksson, R.A., Albrektsson, T., Magnusson, B.: Assessment of bone viability after heat trauma. A histological, histochemical and vital microscopic study in the rabbit. Scand J Plast Reconstr Surg. 1984; 18: 261-268.

26. Fourney, D.R., Schomer, D.F., Nader, R., et al.: Percutaneous vertebroplasty and kyphoplasty for painful vertebral body fractures in cancer patients. J Neurosurg Spine. 2003; 98 : 21-30.

27. Fribourg, D., Tang, C., Sra, P., et al.: Incidence of subsequent vertebral fracture after kyphoplasty. Spine. 2004; 29 : 2270-2276.

28. Gaitanis, I.N., Hadjipavlou, A.G., Katonis, P.G., et al.: Balloon kyphoplasty for the treatment of pathological vertebral compressive fractures. Eur Spine J. 2004; Pub Online: 10.1007/s00586-004-0767-4.

29. Galibert, P., Deramond, H., Rosat, P., Le Gars, D.: Note préliminaire sur le traitment des angiomes vértebraux par vertébroplastie acrylique percutaneé. Neurochirurgie 1987; 33 : 166-168.

30. Gangi, A., Guth, S., Imbert, J.P., et al.: Percutaneous vertebroplasty: indications, technique, and results. Radiographics. 2003; 23: e10.

31. Gangi, A., Kastler, B.A., Dietemann, J.L.: Percutaneous vertebroplasty guided by a combination of CT and fluoroscopy. Am J Neuroradiol. 1994; 15: 83-86.

32. Garfin, S.R., Lin, G., Lieberman, I., et al.: Retropective analysis of the outcomes of balloom kyphoplasty to treat vertebral body compression fracture (VCF) refractory to medical management. Eur Spine J 2001; 10 (suppl): S7.

33. Garfin, S.R., Reilley, M.A.: Minimally invasive treatment of osteoporotic vertebral body compression fractures. Spine J 2002; 2: 76-80.

34. Garfin, S.R., Yuan, H.A., Reiley, M.A.: New technologies in spine: kyphoplasty and vertebroplasty for the treatment of painful osteoporotic compression fractures. Spine. 2001; 26: 1511-1515.

35. Gaughen, J.R. Jr., Jensen M.E., Schweickert, P.A., Kaufmann, T.J., Marx, W.F., Kallmes, D.F.: Relevance of antecedent venography in percutaneous vertebroplasty for the treatment of osteoporotic compression fractures. Am J Neuroradiol. 2002; 23: 594-600.

36. Grados, F., Depriester, C., Cayrolle, G.,et al.: Longterm observations of vertebral osteoporotic fractures treated by percutaneous vertebroplasty. Rheumatology (Oxford). 2000; 39: 1410-1414.

37. Groen, R.J., du Toit, D.F., Phillips, F.M., et al.: Anatomical and pathological considerations in percutaneous vertebroplasty and kyphoplasty: a reappraisal of the vertebral venous system. Spine. 2004; 29: 1465-1471.

38. Hardouin, P., Fayada, P., Leclet, H., Chopin, D.: Kyphoplasty. Joint Bone Spine. 2002; 69: 256-261:

39. Hardouin, P., Grados, F., Cotten, A., Cortet, B.: Should percutaneous vertebroplasty be used to treat osteoporotic fractures? An update. Joint Bone Spine. 2001; 68: 216-221.

40. Harrington, K.D.: Major neurological complications following percutaneous vertebroplasty with polymethylmethacrylate: a case report. J Bone Joint Surg Am. 2001; 83A(7): 1070-1073.

41. Harrop, J.S., Prpa, B., Reinhardt, M.K., Lieberman, I.: Primary and secondary osteoporosis' incidence of subsequent vertebral compression fractures after kyphoplasty. Spine. 2004; 29: 2120-2125.

42. Heini, P.F., Orler, R.: Kyphoplasty for treatment of osteoporotic vertebral fractures. Eur Spine J. 2004; 13: 184192.

43. Heini, P.F., Wälchli, B., Berlemann, U.: Percutaneous transpedicular vertebroplasty with PMMA: operative technique and early results. Eur Spine J 2000; 9: 445-450.

44. Hide, I.G., Gangi, A.: Percutaneous vertebroplasty: history, technique and current perspectives. Clin Radiol. 2004; 59: 461-467.

45. Higgins, K.B., Harten, R.D., Langrana, N.A., Reiter, M.F.: Biomechanical effects of unipedicular vertebroplasty on intact vertebrae. Spine. 2003; 28: 1540-1547.

46. Ide, C., Gangi, A., Rimmelin, A., et al.: Vertebral haemangiomas with spinal cord compression: the place of preoperative percutaneous vertebroplasty with methyl methacrylate. 
Neuroradiology. 1996; 38: 585-589.

47. Jasper, L.E., Deramond, H., Mathis, J.M., Belkoff, S.M.: Material properties of various cements for use with vertebroplasty. J Mater Sci Mater Med. 2002; 13: 1-5.

48. Jensenm M,E, Evansm A,J, Mathism A,J, et al.: Percutaneous polymethylmetacrylate vertebroplasty in the treatment of osteoporotic vertebral body compression fractures: technical aspects. Am J Neuroradiol 1997; 18: 1897-1904.

49. Kim, S.H., Kang, H.S., Choi, J.A., Ahn, J.M.: Risk factors of new compression fractures in adjacent vertebrae after percutaneous vertebroplasty. Acta Radiol. 2004; 45: 440-445.

50. Klimo, P., Jr., Schmidt, M.H.: Surgical management of spinal metastases. Oncologist. 2004; 9: 188-196.

51. Lane, J.M., Girardi, F., Parvaianen, H., et al.. Preliminary outcomes of the first 226 consecutive kyphoplasties for the fixation of painful osteoporotic vertebral compression fractures. Osteoporosis Int 2000;11 (Suppl): S 206.

52. Lane, J.M., Hong, R., Koob, J., et al.: Kyphoplasty enhances function and structural alignment in multiple myeloma. Clin Orthop. 2004; 426: 49-53.

53. Lapras, C., Mottolese, C., Deruty, R., et al.: Percutaneous injection of methyl-metacrylate in osteoporosis and severe vertebral osteolysis. Galibert's technic. Ann Chir. 1989; 43: 371-376.

54. Laredo, I.D., Hamze, B.: Complications of percutaneous vertebroplasty and their prevention. Skeletal Radiol. 2004; 33: 493-505.

55. Ledlie, J.T., Renfro, M.: Balloon kyphoplasty: oneyear outcomes in vertebral body height restoration, chronic pain, and activity levels. J Neurosurg Spine. 2003; 98: 36-42.

56. Leidig, G., Minne, H.W., Sauer, P., et al.: A study of complaints and their relation to vertebral destruction in patients with osteoporosis. Bone Miner. 1990; 8: 217-129.

57. Leidig, G., Minne, H.W., Schlaich, C., et al.: Clinical grading of spinal osteoporosis: quality of life components and spinal deformity in women with chronic low back pain and women with vertebral osteoporosis. J Bone Miner Res. 1997; 12: 663-675.

58. Levine, S.A., Perin, L.A., Hayes, D., Hayes, W.S.: An evidence-based evaluation of percutaneous vertebroplasty. Manag Care. 2000; 9: 56-60.

59. Lieberman, I.H., Dudeney, S., Reinhardt, M.K., Bell, G.: Initial outcome and efficacy of "kyphoplasty" in the treatment of painful osteoporotic vertebral compression fractures. Spine. 2001 Jul 15; 26: 1631-1638.

60. Lieberman, I., Reinhardt, M.K.: Vertebroplasty and kyphoplasty for osteolytic vertebral collapse. Clin Orthop. 200; 415(Suppl): S176-186.

61. Lin, E.P., Ekholm, S., Hiwatashi, A., Westesson, P.L.: Vertebroplasty: cement leakage into the disc increases the risk of new fracture of adjacent vertebral body. Am J Neuroradiol. 2004; 25: 175-180.

62. Lopes, N.M., Lopes, V.K.: Paraplegia complicating percutaneous vertebroplasty for osteoporotic vertebral fracture: case report. Arq Neuropsiquiatr. 2004; 62: 879-881.

63. Martin, J.B., Jean, B., Sugui, K., et al.: Vertebroplasty: clinical experience and folow-up results. Bone 1999; 25 (2 Suppl): 11S-15S

64. Martínez Quiñones, J.V., Hernández, G.; Percutaneous vertebroplasty: technique and early results in 25 procedures. Neurocirugía. 2003; 14: 323-332.

65. Masala S, Cesaroni A, Sergiacomi G, et al. Percutaneous kyphoplasty: new treatment for painful vertebral body fractures. In Vivo. 2004;18:149-153.

66. Masala, S., Fiori, R., Massari, F., Cantonetti, M., Postorino, M., Simonetti, G.: Percutaneous kyphoplasty: indications and technique in the treatment of vertebral fractures from myeloma. Tumori. 2004; 90: 22-26.

67. Mathis, J.M., Petri, M., Naff, N.: Percutaneous vertebroplasty treatment of steroid-induced osteoporotic compression fractures. Arthritis Rheum. 1998; 41: 171-175.

68. Mathis, J.M., Ortiz, O., Zoarski, G.H.: Vertebroplasty versus kyphoplasty: a comparison and contrast. AJNR Am J Neuroradiol 2004; 25: 840-845.

69. Maynard, A.S., Jensen, M.E., Schweickert, P.A., et al.: Value of bone scan imaging in predicting pain relief from percutaneous vertebroplasty in osteoporotic vertebral fractures. AJNR Am J Neuroradiol. 2000; 21: 1807-1812.

70. McGraw, J.K., Heatwole, E.V., Strnad, B.T., Silber, J.S., Patzilk, S.B., Boorstein, J.M.: Predictive value of intraosseous venography before percutaneous vertebroplasty. J Vasc Interv Radiol. 2002; 13: 149-153.

71. Mehdizade, A., Lovblad, K.O., Wilhelm, K.E., et al.: Radiation dose in vertebroplasty. Neuroradiology. 2004; 46: 243-245.

72. Myers, M.E.: Vertebroplasty and kyphoplasty: is one of these procedures the best choice for all patientes? Am J Neuroradiol. 2004; 25: 1297.

73. Olan, W.J.: Kyphoplasty: Balloon-assisted vertebroplasty. ASNR Spine Symposium. Vancouver, BC 2002; pp: 115-117.

74. Padovani, B., Kasriel, O., Brunner, P., Peretti-Viton, P.: Pulmonary embolism caused by acrylic cement: a rare complication of percutaneous vertebroplasty. Am J Neuroradiol. 1999; 20: 375-357.

75. Perisinakis, K., Damilakis, J., Theocharopoulos, N., et al.: Patient exposure and associated radiation risks from fluoroscopically guided vertebroplasty or kyphoplasty. Radiology. 2004; 232: 701-707.

76. Perrin, C., Jullien, V., Padovani, B., Blaive, B.: Percutaneous vertebroplasty complicated by pulmonary embolus of acrylic cement. Rev Mal Respir. 1999; 16: 215-217.

77. Philips, F.M.: Minimally invasive treatments of osteoporotic vertebral compression fractures (suppl). Spine 2003; 28: S45-S53.

78. Phillips, F.M., Ho, E., Campbell-Hupp, M., McNally, 
T., Todd Wetzel, F., Gupta, P.: Early radiographic and clinical resuits of balloon kyphoplasty for the treatment of osteoporotic vertebral compression fractures. Spine. 2003;28:22602265 .

79. Phillips, F.M., Pfeifer, B.A., Lieberman, I.H., et al.: Minimally invasive treatments of osteoporotic vertebral compression fractures: vertebroplasty and kyphoplasty. Instr Course Lect. 2003; S2: 559-567.

80. Phillips, F.M., Todd Wetzel, F., Lieberman, I., Campbell-Hupp, M.: An in vivo comparison of the potential for extravertebral cement leak after vertebroplasty and kyphoplasty. Spine. 2002; 27: 2173-2178.

81. Provenzano, M.J., Murphy, K.P., Riley, L.H.: 3rd. Bone cements: review of their physiochemical and biochemical properties in percutaneous vertebroplasty. Am J Neuroradiol. 2004; 25: 1286-1290.

82. Rami, P.M., McGraw, J.K., Heatwole, E.V., Boorstein, J.M.: Percutaneous vertebroplasty in the treatment of vertebral body compression fracture secondary to osteogenesis imperfecta. Skeletal Radiol. 2002; 31: 162-165. Erratum in: Skeletal Radio 2002; 31: 558.

83. Ratliff, J., Nguyen, T., Heiss, J.: Root and spinal cord compression from methylmethacrylate vertebroplasty. Spine 2001;26: E300-2002.

84. Ross, P.D., Ettinger, B., Davis, J.W., et al.: Evaluation of adverse health outcomes associated with vertebral fractures. Osteoporos Int. 1991; 1: 134-140.

85. Schlaich, C., Minne, H.W., Bruckner, T.: Reduced pulmonary function in patients with spinal osteoporotic fractures. Osteoporosis Int 1998; 8: 261-267.

86. Seibert, J.A.: Vertebroplasty and kyphoplasty: do fluoroscopy operators know about radiation dose, and should they want to know? Radiology. 2004; 232: 633-634.

87. Silman, A.J., y el EVOS Study Group The European Vertebral Osteoporosis Study Group. (EVOS). En: Christiansen C, Riis B, eds. Osteoporosis. Proceedings of The Fourth International Symposium on Osteoporosis and Consensus Development Conference, Rodovre, 1993; pp: 65-66.

88. Silvermann, S.L.: The clinical consequences of vertebral compression fractures. Bone 1992; 13 Suppl: 23-25.

89. Sosa, M.: La fractura vertebral: una entidad en busca de definición. Med Clin (Barc) 2000; 115: 661-662.

90. Teng, M.M., Wei, C.J., Wei, L.C., et al.: Kyphosis correction and height restoration effects of percutaneous vertebroplasty. Am J Neuroradiol 2003; 24: 1893-1900.

91. Theodorou, D.J., Theodorou, S.J., Duncan, T.D., Garfin, S.R., Wong, W.H.: Percutaneous balloon kyphoplasty for the correction of spinal deformity in painful vertebral body compression fractures. Clin Imaging. 2002; 26: 1-5.

92. Thongtrangan, I., Le, H., Park, J., Kim, D.H.: Minimally invasive spinal surgery: a historical perspective. Neurosurg
Focus. 2004; 16: E 13.

93. Tomita, S., Molloy, S., Jasper, L.E., et al.: Biomechanical comparison of kyphoplasty with different bone cements. Spine. 2004; 29: 1203-1207.

94. Truumees E, Hilibrand A, Vaccaro AR. Percutaneous vertebral augmentation. Spine J. 2004;4:218-229.

95. Uppin, A.A., Hirsch, J.A., Centenera, L.V., Pfiefer, B.A., Pazianos, A.G., Choi, I.S.: Occurrence of new vertebral body fracture after percutaneous vertebroplasty in patients with osteoporosis. Radiology. 2003; 226: 119-124.

96. Vasconcelos, C., Gailloud, P., Beauchamp, N.J., et al.: Is percutaneous vertebroplasty without pretreatment venography safe? Evaluation of 205 consecutives procedures. AJNR Am J Neuroradiol. 2002; 23: 913-917.

97. Watts, N.B., Harris, S.T., Genant, H.K.: Treatment of painful osteoporotic vertebral fractures with percutaneous vertebroplasty or kyphoplasty. Osteoporos Int. 2001; 12: 429437.

98. Wenger, M., Braun, M., Markwalder, T.M.: Vertebroplasty and kyphoplasty: a review. Rev Med Suisse Romande, 2002; 122: 557-560.

99. Wenger, M., Markwalder, T,M.: Surgically controlled, transpedicular methyl methacrylate vertebroplasty with fluoroscopic guidance, Acta Neurochir 1999; 141: 625-631.

100. Weill, A., Chiras, J., Simon, et al.: Spinal metastases: indications for and resuits of percutaneous injection of acrylic surgical cement. Radiology 1996; 199: 241-247.

101. Wilcox, R.K.: The biomechanics of vertebroplasty: a review. Proc Instn Mech Engrs 2004; 218 (part H):1-10.

102. Wilson, D.R., Myers, E.R., Mathis, J.M., et al.: Effect of augmentation on the mechanics of vertebral wedge fractures. Spine 2000; 25: 158-165.

103. Wu, S.S., Lachmann, E., Nagler, W.: Current medical, rehabilitation, and surgical management of vertebral compression fractures. J Womens Health (Larchmt).2003; 12: 17-26.

104. Yoo, K.Y., Jeong, S.W., Yoon, W., Lee, J.: Acute respiratory distress syndrome associated with pulmonary cement embolism following percutaneous vertebroplasty with polymethylmethacrylate. Spine. 2004; 29: E294-297.

105. Zoarski, G.H., Snow, P., Olan, W.J., et al.: Percutaneous vertebroplasty for osteoporotic compression fractures: quantitative prospective evaluation of long-term outcomes. J Vasc Interv Radiol. 2002; 13 (2 Pt 1):139-148.

Martínez-Quiñones, J.V.; Aso Escario, J.; Arregui Calvo, R.: Refuerzo vertebral percutáneo: vertebroplastia y cifoplastia. Procedimiento técnico. Neurocirugía 2005; 16: 427-440.

Correspondencia postal: Dr. D. José Vicente Martínez Quiñones. M. Antonia de Oviedo, 1; portal 10, 7º-B. 50008 Zaragoza. 